\title{
Effect of Chitosan Magnetic Nanoparticles Loaded with Ang2-siRNA Plasmids on the Growth of Melanoma Xenografts in Nude Mice
}

This article was published in the following Dove Press journal: Cancer Management and Research

\author{
Xiuying Shan ${ }^{1, *}$ \\ Wenjun $\mathrm{Yu}^{\mathrm{l}}{ }^{*}$ \\ Xuejun $\mathrm{Ni}^{1}{ }^{1} *$ \\ Tingting $\mathrm{Xu}^{\prime}$ \\ Chen Lei ${ }^{1}$ \\ Zhaoliang Liu' \\ Xuefeng $\mathrm{Hu}^{2}$ \\ Yanding Zhang ${ }^{2}$ \\ Beichen Cai' \\ Biao Wang'
}

'Department of Plastic Surgery, The First Affiliated Hospital of Fujian Medical University, Fuzhou 350005, People's Republic of China; ${ }^{2}$ College of Life Sciences, Fujian Normal University, Fuzhou 350108, People's Republic of China

*These authors contributed equally to this work
Correspondence: Biao Wang

Email wangbiao@fjmu.edu.cn
Purpose: Angiopoietin-2 (Ang-2) has been proven to be a potential agent for malignant cancer treatment. The aim of the current study was to investigate the inhibitory effects of chitosan magnetic nanoparticles (CMNPs) loaded with Ang-2 small interfering RNA (Ang2siRNA) plasmids (Ang2-CMNPs) on malignant melanoma.

Materials and Methods: Melanoma-bearing nude mice were treated with Ang2-CMNPs and control CMNPs. Tumor volumes in each group were recorded. Real-time fluorescence quantitative-PCR was used to measure the relative Ang-2gene expression. Angiogenesis and Ang-2 expression in tumors were measured by immunohistochemistry. Cell apoptosis in each group was measured by TUNEL staining, and the expression of Bax, Bcl-2 and cleaved caspase- 3 was analyzed by immunohistochemistry.

Results: The progression of melanoma was significantly inhibited by Ang2-CMNP treatment. Ang2-CMNP treatment efficiently inhibited tumor growth and in-situ Ang-2 expression compared with those of the control group. Furthermore, Ang2-CMNP treatment significantly inhibited tumor angiogenesis and promoted cell apoptosis by regulating the Bax/Bcl-2 ratio and increasing cleaved caspase-3 expression in vivo.

Conclusion: In summary, Ang2-CMNP treatment increased the regression of normalappearing vessels in the tumor microenvironment and induced the melanoma cells apoptosis through the mitochondrial apoptotic pathway, suggesting the potential clinical use of Ang2CMNPs in malignant melanoma treatment.

Keywords: angiopoietin-2, small interfering RNA, malignant melanoma, chitosan magnetic nanoparticles, apoptosis

\section{Introduction}

Malignant melanoma, one of the most aggressive cancers, was the fifth most common malignancy in 2019. ${ }^{1}$ Recent studies on antiangiogenic and immune checkpoint therapies have provided promising results for melanoma inhibition., ${ }^{2,3}$ However, questions remain regarding how to decrease systemic side effects and tumor resistance to treatment.

Tumor angiogenesis, the crucial factor in cancer growth and progression, is mainly regulated by vascular endothelial growth factor A (VEGFA). ${ }^{4,5}$ The VEGFA inhibitor bevacizumab, the first antiangiogenic drug, was approved by the US Food and Drug Administration in 2004 and recommended in combination with chemotherapy to treat several cancer types. ${ }^{5-7}$ However, the combination of VEGFA inhibitors with first-line anticancer drugs generally produced modest survival improvements. ${ }^{5,8}$ Increasing 
evidence indicates that the mechanism underlying the limited clinical efficacy could be attributed to stimulating compensatory proangiogenic signaling. ${ }^{9,10}$

Angiopoietin-2 (Ang-2), a ligand of tyrosine kinase with immunoglobulin-like and epidermal growth factor homology domains (Tie-2), is a critical proangiogenic factor that sustains tumor angiogenesis, which has made it a potential target for anticancer therapy. ${ }^{11-13}$ Ang-2 blockade has been proven to inhibit sustained growth and angiogenesis in various tumor models, ${ }^{14,15}$ including a spontaneous islet tumor model known to be resistant to VEGFA inhibitors. ${ }^{10}$ The main treatment-related side effects of the anti-Ang2 monoclonal antibody nesvacumab in patients with advanced tumors included fatigue, peripheral edema, diarrhea and decreased appetite; side effects and the pharmacokinetics (PK) profile were dose-dependent, indicating that repeat dosing was needed to maintain Ang-2 levels. ${ }^{16}$ Therefore, a strategy to decrease the Ang-2 dosage in situ in a safe, persistent and efficient manner would be highly desirable for melanoma treatment. To address these concerns, gene-based therapy and magnetic target treatment can be combined to improve therapeutic efficacy.

Recently, chitosan has been shown to be a promising carrier for specific, targeted drug delivery systems to treat cancers and has applications in tissue engineering. ${ }^{17,18}$ In our previous study, we engineered CMNPs containing Ang-2 small interfering RNA (Ang2-siRNA) plasmids, ${ }^{19}$ which are made from a magnetic targeted drug delivery system (MTDDS). ${ }^{20}$ The MTDDS, in combination with a stable external magnetic field, allows for the delivery of particles to the chosen target area and retrains the particles at the local site while the medication is released and directly acts on the target site. ${ }^{20}$ The aim of our current study was to evaluate the effects of Ang2-CMNPs on malignant melanoma growth, apoptosis and angiogenesis, and to clarify the mechanisms of the antitumor effect of Ang2-CMNPs using a nude mouse model of malignant melanoma.

\section{Materials and Methods}

\section{Animals}

The nude mice (male, BALB/c, specific-pathogen-free breed) were purchased from the Shanghai Experimental Animal Center. All the experiments as performed in accordance with the "Guide for the Care and Use of Laboratory Animals" from the National Institutes of Health (NIH), as well as the research approach gaining approval from the Institutional Animal Care and Use Committee of the First
Affiliated Hospital of Fujian Medical University. Each mouse was maintained in a SPF sterile laminar flow chamber, with free access to water and food, and was allowed for 1 week of adaptive feeding at $24^{\circ} \mathrm{C}$ with a normal light cycle prior to experimental surgery to minimize animal suffering.

\section{Ang2-CMNP Preparation}

The Ang2-siRNA-loaded and chitosan-coated magnetic nanoparticles were preserved by our laboratory and prepared as previously reported; ${ }^{19}$ the particle sizes ranged from 55.2 $\mathrm{nm}$ to $82.5 \mathrm{~nm}$, with a mean diameter of $67 \mathrm{~nm}$, and the magnetic nanoparticles also exhibited good magnetic responsivity. The pGenesil-3 recombinant plasmid, pGenesil-3 bacterial culture, 1 plus 2 pGenesil-3 Ang2-siRNA recombinant plasmid, and 1 plus 2 pGenesil-3 Ang2-siRNA bacterial culture were all purchased at Wuhan Xima Biotechnology Co., Ltd. In addition, the 1 plus 2 pGenesil-3 Ang2-siRNA (also known as the Ang2-siRNA plasmid) contained the following 2 RNAi sequences:

S1: 5'-ACCCCACTGTTGCTAAAGATTCAAGAGA TCTTTAGCAACAGTGGGGTTTTTT-3';

S2: 5'-GCCACGGTGAATAATTCAGTTCTCGAGA ACTGAATTATTCACCGTGGCTTTTT-3'.

\section{Tumor Xenografts in Nude Mice}

Human malignant melanoma A375 cells were purchased from Biochemistry and Cell Biology, Shanghai Institutes for Biological Sciences (Shanghai, China). Cells in the logarithmic growth phase were collected by dissociated with $0.25 \%$ trypsin and washed twice with serum-free DMEM. Then, a cell suspension with a concentration of $5 \times 10^{7}$ cells $/ \mathrm{mL}$ was prepared. $100 \mu \mathrm{L}$ suspension was injected into the right axillary subcutaneous tissue of the nude mice. The tumor status and health of the nude mice were observed. The mice were randomly divided into two groups (10 mice/group) when the subcutaneous tumors grew to approximately $6 \mathrm{~mm} \times 6 \mathrm{~mm} \times 3 \mathrm{~mm}$. Additionally, a 4000 GS magnetic field was applied to the right axillary subcutaneous tissue of each mouse, and $60 \mathrm{~min}$ later, the magnetic field was removed. In the control group, empty magnetic nanoparticles were injected into the mice via the tail vein, while the experimental group was injected with an equal volume of Ang2CMNPs. This injection was administered every 3 days, 8 times in total. During the experiment, the tumor length (L) and width (W) were measured with a Vernier caliper. The tumor volume was calculated with the formula: $\mathrm{V}=$ $\mathrm{L}^{2} \times \mathrm{W}^{21}$. The calculated results were used to plot the 
tumor growth curve. The tumor characteristics were observed by HE and Prussian blue staining and were photographed.

\section{Microvessel Density Evaluation}

The xenografted tumor tissues of the nude mice were cut into appropriate sizes, fixed with $4 \%$ paraformaldehyde for 16-24 hours, embedded with paraffin, and cut into $4 \mu \mathrm{m}$ sections. Positive CD34 staining is shown in tan. According to the Weidner correction method, any antibody staining of a single endothelial cell or cell mass is considered countable in the capillaries if the lumen is formed or if there is a clear boundary between the surrounding microvessels, tumor cells and other connective tissue. The microvessels in the parenchyma of the tumor and in the soft tissue at the junction of the tumor were excluded. The vessels within the smooth muscle wall or with a lumen diameter greater than 8 red blood cells were also excluded. Tumor microvessel density (MVD) was examined by searching for the best stained area using a low magnification lens and then changing it into a high magnification lens to select three different visual fields and calculate the average.

\section{The Expression Level of Ang2 mRNA}

TRIzol reagent (Takara, Dalian, Liaoning Province, China) was used to extract total RNA from the transplanted malignant melanomas of the nude mice in the experimental and control groups. The extracted RNA $(1.0 \mu \mathrm{g})$ was used for cDNA synthesis using the Prime Script RT Reagent Kit (TAKARA). Quantitative real-time PCR was performed using the SYBR Premix Ex $\mathrm{Taq}^{\mathrm{TM}}$ Kit (TAKARA) in a LightCycler 480 (Roche, Basel, Switzerland) under the following conditions: 35 cycles at $94^{\circ} \mathrm{C}$ for $30 \mathrm{~s}, 60^{\circ} \mathrm{C}$ for $30 \mathrm{~s}$, and $72^{\circ} \mathrm{C}$ for $30 \mathrm{~s}$. The corresponding $\mathrm{Ct}$ values were recorded and Ang2 expression was calculated using the $2^{-\Delta \Delta} \mathrm{Ct}$ method.

\section{Assessment of the Apoptosis Rate}

After the samples were dehydrated with an ethanol gradient $(100 \%, 95 \%, 85 \%, 70 \%$, and $50 \%)$, washed with PBS and fixed with 4\% paraformaldehyde for 15 minutes at room temperature, the prepared paraffin-embedded tissues were cut, deparaffinized, and washed with xylene solution (two times each for 5 minutes). The excess liquid was removed from the slides, and then $100 \mu \mathrm{L}$ of newly prepared protease $\mathrm{K}$ working solution $(20 \mu \mathrm{g} / \mathrm{mL})$ was added to each slide and incubated for $10-30 \mathrm{~min}$ at
A

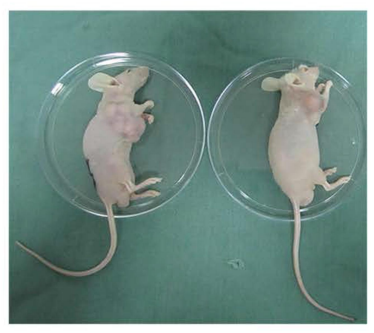

B

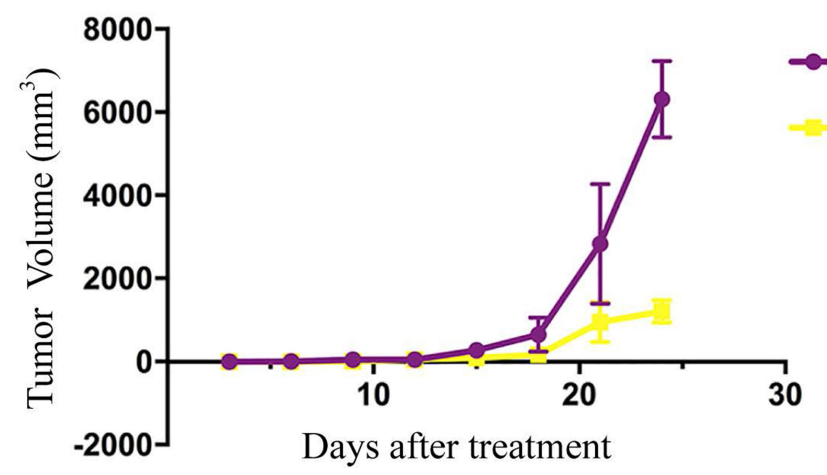

Ang2-CMNPs
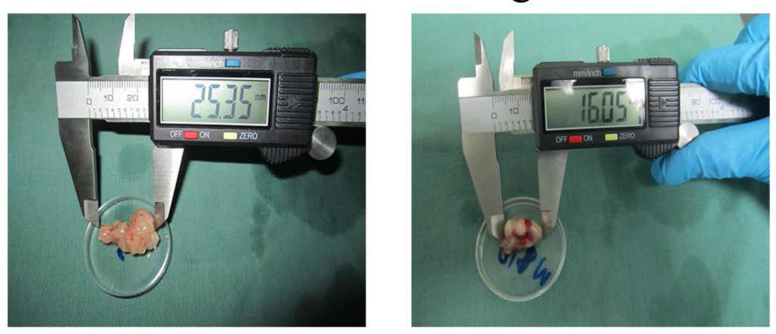

Control

Ang2-CMNPs

Figure I Effect of Ang2-CMNPs on tumor growth in malignant melanoma-bearing nude mice. (A) Representative images show the inhibition of tumor growth in the groups treated with Ang2-CMNPs and empty magnetic nanoparticles, with tumor volume observed by day 24. (B) The tumor volume was evaluated every 3 days posttransplantation. Ang2-CMNP treatment significantly decreased tumor volume compared to that of the control group, which was treated with empty magnetic nanoparticles. Analyses were performed every 3 days. 
A

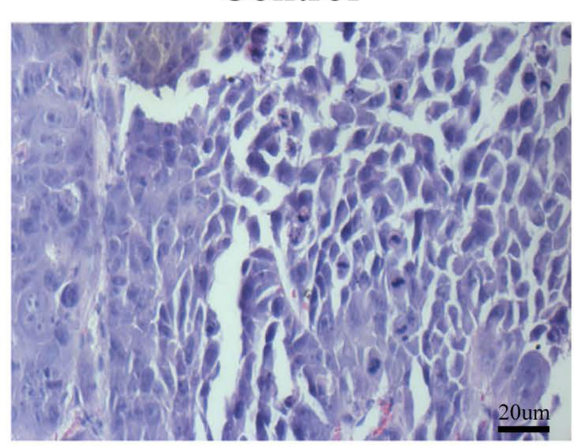

B

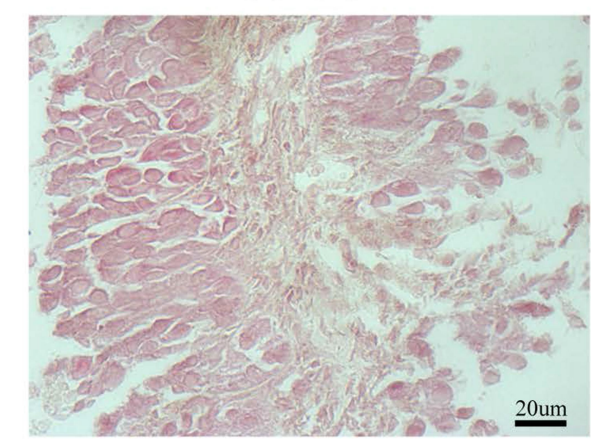

Ang2-CMNPs

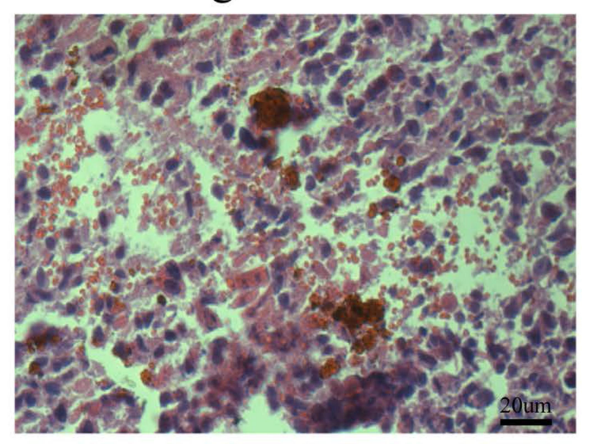

Ang2-CMNPs

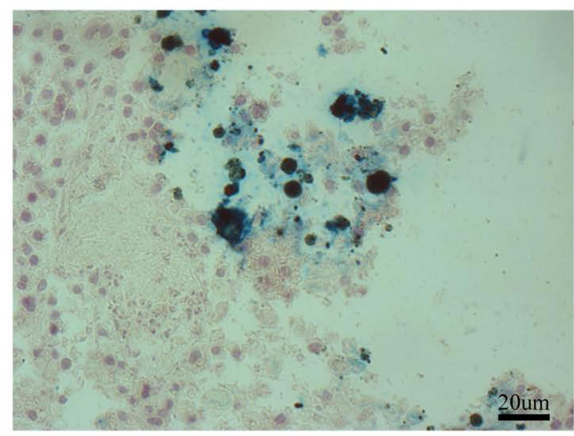

Figure 2 Histological analysis of tumor tissues posttreatment. (A) HE staining showed increased nanoparticles in tumor tissues treated with Ang2-CMNPs compared with control tissues treated with empty magnetic nanoparticles (400x). (B) The Ang2-CMNP-treated group was strongly positive (dark blue spots, Prussian blue staining), whereas the empty magnetic nanoparticle-treated group was weakly positive (light blue spots, Prussian blue staining; 400×).

room temperature. Then, the slides were washed with PBS, fixed with 4\% formaldehydum polymerisatum for $5 \mathrm{~min}$, and washed with PBS again. Then, $100 \mu \mathrm{L}$ of equilibration buffer was added. After 5-10 min of incubation at room temperature, $100 \mu \mathrm{L}$ of TdT enzyme reaction solution was added. The slides were placed into an incubation box with an end-labeling reaction for 60 minutes at room temperature. Then, the slides were placed into a $20 \times \mathrm{SSC}$ solution to stop the reaction. The slides were incubated for $15 \mathrm{~min}$ at room temperature and washed with PBS, and $100 \mu \mathrm{L}$ DAB solution was added until the color of the background turned light brown. The slides were mounted and observed with a microscope.

\section{Immunohistochemical Detection of Ang-2, Bax, Bcl-2 and Cleaved Caspase-3 Expression in Tissues}

The sections were prepared and deparaffinized in xylene and dehydrated through a graded series of alcohols. Highpressure antigen retrieval was performed with citrate antigen repair solution, and the sections were then incubated in 3\% hydrogen peroxide at room temperature for $20 \mathrm{~min}$. The slices were incubated with primary rabbit polyclonal antibodies against Ang-2, Bax, Bcl-2 and cleaved caspase 3 (Abcam, Cambridge, MA) at $4^{\circ} \mathrm{C}$ overnight and then incubated with biotin-labeled secondary antibodies at $37^{\circ} \mathrm{C}$ for 30 min. Next, 3,3'-diaminobenzidine (DAB) was added and incubated at room temperature for $10 \mathrm{~min}$ and the sections were then stained with hematoxylin at room temperature for $2 \mathrm{~min}$. Finally, the slides were gently washed with deionized water, dehydrated by gradient alcohol, mounted in neutral balsam, and observed using an optical microscope. Immunopositivity was scored by image J (version 1.50, NIH, https://imagej.nih.gov/ij/, Bethesda, MD, USA). Negative staining was evaluated as follows: 1, no staining; 2 , low positive staining; 3 , positive staining and 4, high positive staining. The final score for each group was determined by the mean of the individual scores.

\section{Statistical Analysis}

All statistical analyses were performed using SPSS software (version 22.0; SPSS, Inc., Chicago, IL, USA). The data are presented as the mean \pm standard deviation. 
A

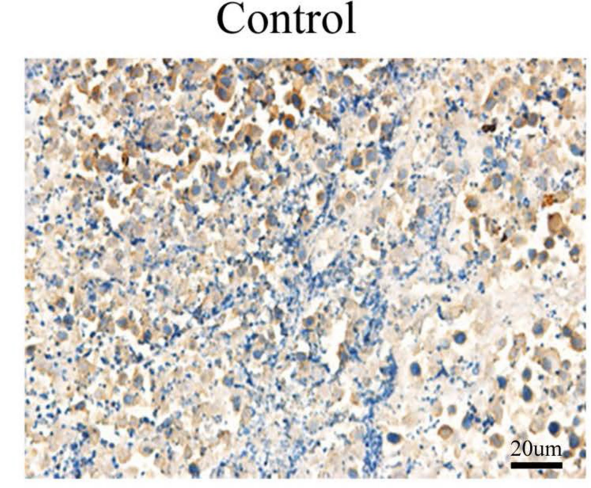

Ang2-CMNPs

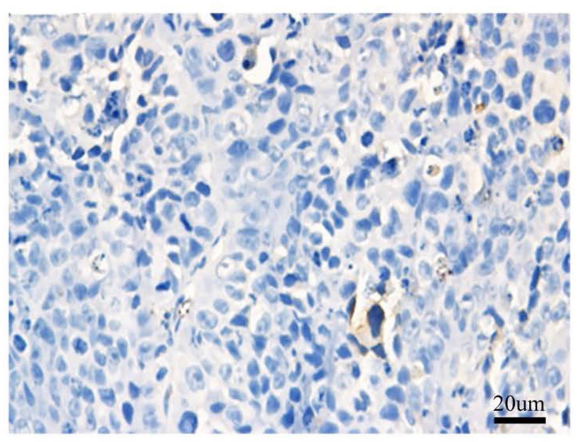

B

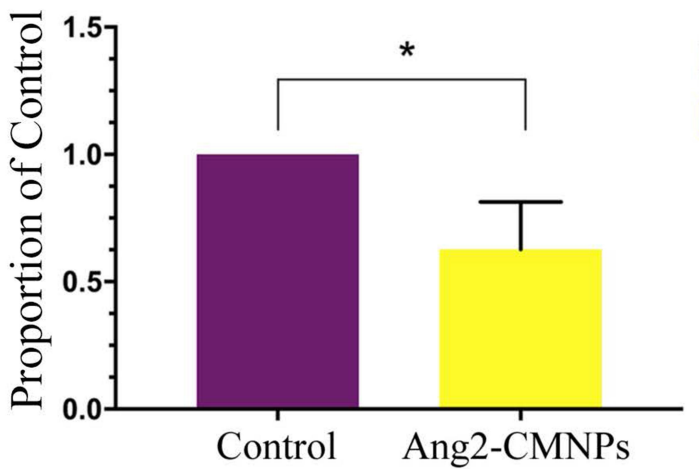

Control

Ang2-CMNPs

Figure 3 In situ Ang2 expression in tumor tissues was further confirmed by qRT-PCR. (A) Immunohistochemical staining of tumor tissue sections shows decreased expression of Ang2 in the Ang2-CMNP-treated group compared with the empty magnetic nanoparticle-treated control group (400x). (B) qRT-PCR showed reduced Ang2 expression in tumor tissues treated with Ang2-CMNPs compared to empty magnetic nanoparticle-treated controls. The values are expressed as the mean \pm SD. $* P<0.05$ compared with the control group. Analyses were performed on day 24 posttreatment using independent-samples t-tests.

Comparisons between two groups were performed using an independent-samples $t$ test. Multiple group comparisons were performed by one-way ANOVA, followed by Tukey's post hoc analysis. $\mathrm{P}<0.05$ was considered to indicate a statistically significant difference.

\section{Results}

\section{Inhibitory Effect of Ang2-CMNP}

\section{Treatment on Tumor Growth in}

\section{Malignant Melanoma-Bearing Nude Mice}

The effect of Ang2-CMNPs on tumor volume and the growth of transplanted malignant melanomas in nude mice was observed for 24 days. Tumors formed in each nude mouse over time, and the tumor volume in the Ang2CMNP group was significantly smaller than that in the control group (Figure 1A). The tumor growth in mice was determined by taking two perpendicular measurements every 3 days (Figure 1B). The average volume of subcutaneous tumors in the Ang2-CMNP group (1200.81 $\mathrm{mm}^{3}$ ) decreased by $80.98 \%$ compared with that of the control group $\left(6312.01 \mathrm{~mm}^{3}\right)(\mathrm{P}<0.05)$.

\section{Ang2-CMNPs Recruited More Nanoparticles to the Tumor Tissue}

Ang2-CMNPs were not found in the tumor tissues of the control group, and Prussian blue staining was negative. However, magnetic nanoparticles were aggregated in the membranes and vessels of the tumor tissues of the experimental group, and Prussian blue staining was strongly positive (Figure 2A-B). These results revealed that under a stable magnetic field, Ang2-CMNPs could be directed to target transplanted malignant melanoma tumor tissues and aggregated in the tumor tissues, suggesting that the stable magnetic field had effective targeting.

\section{Decreased Ang2 Production in vivo Led to Reduced Angiogenesis}

Real-time fluorescence quantitative RT-PCR was used to measure the relative Ang2 gene expression. The mRNA level of Ang2 in the Ang2-CMNP group was significantly reduced to approximately $59.6 \%$ compared with that of the control group (Figure 3A-B) $(\mathrm{P}<0.01)$. 

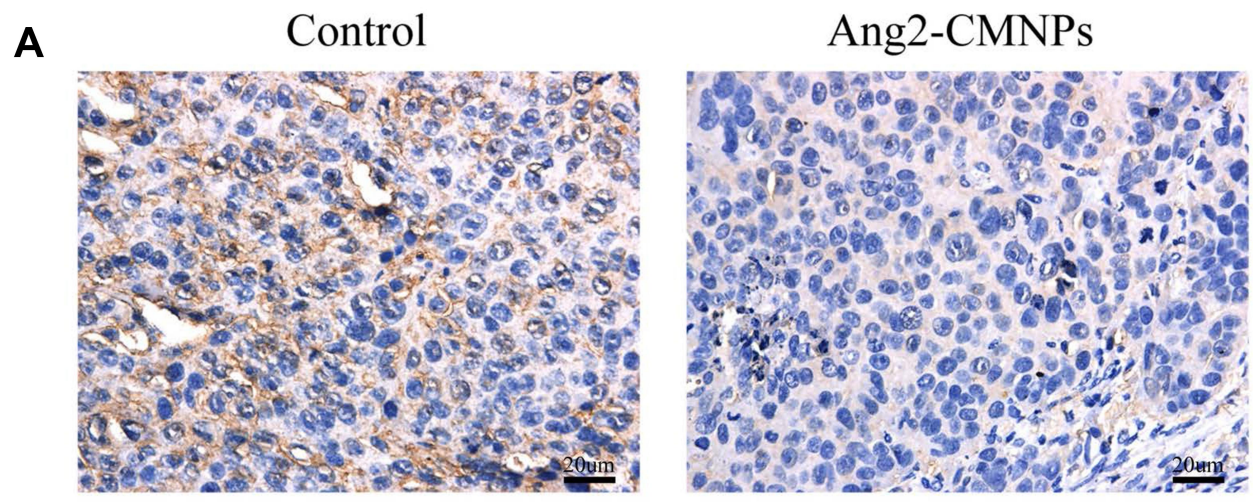

B

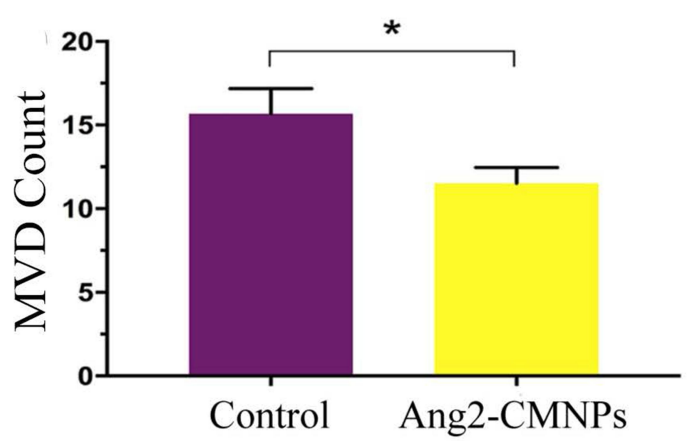

Control

Ang2-CMNPs

Figure 4 Evaluation of blood vessel formation in each group. (A) CD34 labeling of endothelial cells shows decreased staining in the tumor tissue of the Ang2-CMNP-treated group compared with the empty magnetic nanoparticle-treated control group (400x). (B) The average microvessel density shows decreased blood vessels in the tumor tissues treated with Ang2-CMNPs compared with empty magnetic nanoparticle-treated controls. *P<0.05 compared with the control group.

CD34 is highly expressed in the cytoplasm of vascular endothelial cells and can be considered one of the markers of tumor microvessel angiogenesis. A CD34 monoclonal antibody was used as a specific marker for newly formed microvessels. The microvessel density (MVD) in malignant melanoma transplanted tumor tissue was determined by an immunohistochemical SP method using a monoclonal antibody against CD34. The immunohistochemical SP results demonstrated that there were many irregular and disordered blood vessels in malignant melanomas (Figure 4A). The MVD of the experimental group was significantly lower than that of the control group (Figure 4B) $(\mathrm{P}<0.05)$.

\section{Ang2-CMNPs Increased Melanoma Cell Apoptosis in vivo and Induced Apoptosis Through the Mitochondria-Mediated}

\section{Pathway}

The apoptosis rate of malignant melanoma tissues in each group was evaluated using the TUNEL assay. The apoptosis rate of malignant melanoma cells in the Ang2-CMNP group was significantly higher than that of the control group
$(\mathrm{P}<0.05)$ (Figure 5A-B).To explore the mechanism by which Ang2-CMNPs induced melanoma cell apoptosis, we examined the expression of related proteins in the mitochondria-mediated apoptosis pathway, including Bax, Bcl-2 and cleaved caspase-3. Ang2-CMNPs significantly increased the expression levels of bax and cleaved caspase- 3 in the tumor tissue in the Ang2-CMNP-treated group compared with the empty magnetic nanoparticle-treated controls $(\mathrm{P}<0.05)$ (Figure 6A and C; Figure 7A-B). In contrast, the levels of Bcl-2 were decreased after treatment with Ang2-CMNPs. A significantly higher $\mathrm{Bax} / \mathrm{Bcl}-2$ ratio was observed in the tumor tissue of the Ang2-CMNP group than that of the control group $(\mathrm{P}<0.05)$ (Figure $6 \mathrm{~B}$ and $\mathrm{C})$.

\section{Discussion}

Malignant melanoma has long been considered one of the most invasive cancers worldwide. Previous studies suggested that patients could benefit from antiangiogenic therapy. In this study, we explored a new strategy to increase the efficacy of Ang-2-based antiangiogenic therapy for malignant melanoma. A combination of Ang-2 siRNA and CMNPs was used to treat melanoma-bearing nude mice. We demonstrated 
A

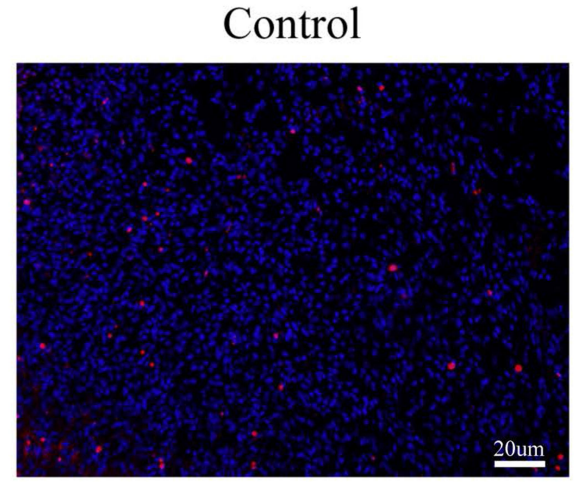

B

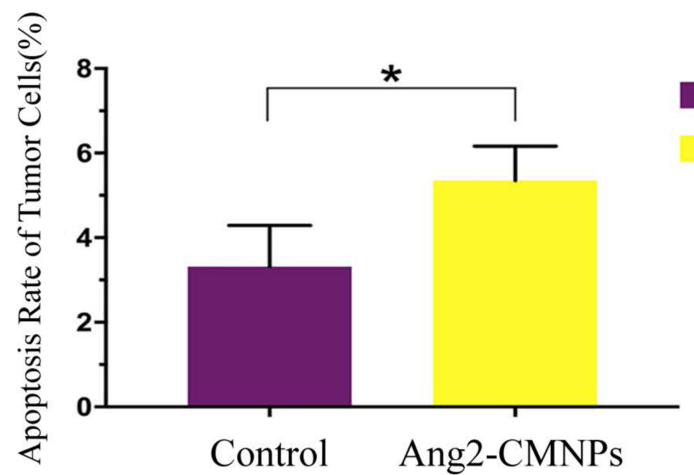

Ang2-CMNPs

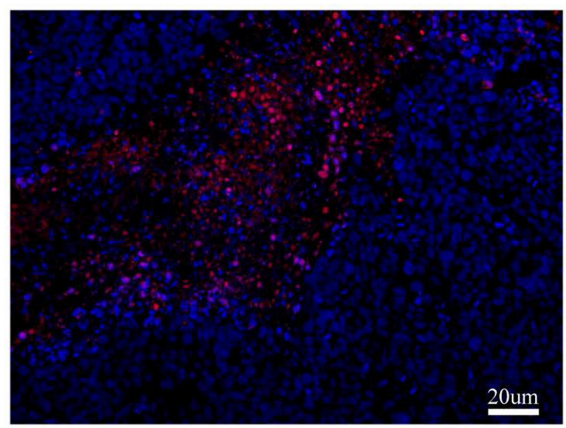

Control

Ang2-CMNPs

Figure 5 Cell apoptosis was evaluated in malignant melanoma tissues. (A) TUNEL staining shows an increase in TUNEL-positive cells in the tumor tissue of the Ang2CMNP-treated group compared with empty magnetic nanoparticle-treated controls $(400 \times)$. (B) The average number of TUNEL-positive cells per field in tissue sections shows increased numbers of apoptotic cells in the Ang2-CMNP-treated group compared with the empty magnetic nanoparticle-treated control group. $* P<0.05$ compared with the control group.

that Ang2-CMNPs significantly inhibited tumor growth in nude mice compared to control mice.

Many proangiogenic cytokine signals have been identified as key regulators in the growth and progression of malignant melanoma. Among these targets, VEGF is one of the most active factors promoting angiogenesis. Downregulation of VEGF through gene therapy may reduce tumor growth and even lead to tumor shrinkage by inhibiting tumor angiogenesis and metastasis. ${ }^{22}$ However, recent studies have shown that tumor revascularization and recurrence often occur after VEGF blockade treatment, and the escape mechanisms are mainly thought to be due to the upregulation of alternative proangiogenic cytokines. ${ }^{11,15}$

Ang-2, which is mainly upregulated in endothelial cells and some malignant cells, is considered to be an important proangiogenic molecule during tumor development and metastasis. $^{23}$ A few publications have demonstrated that increased levels of Ang-2 predict unfavorable clinical outcomes in patients treated with bevacizumab in combination with chemotherapy. ${ }^{24,25}$ Recently, a strong relationship between serum Ang-2 and resistance to immune checkpoint therapy has been reported. ${ }^{26}$ Ang-2 enhanced the proangiogenic and immunosuppressive activities in the tumor microenvironment by increasing PD-L1 expression on M2-polarized macrophages. ${ }^{26}$ Taken together, these findings highlight the potential therapeutic value of specific Ang2 inhibitors, some of which are currently being verified in clinical trials. Although specific Ang-2 inhibitors have shown efficacy in several tumor models, safety concerns, such as systemic side effects, have attracted increasing attention. $^{15,16,25}$ Thus, it is important to develop safe gene delivery approaches for clinical trials.

To address these limitations, chitosan magnetic nanoparticles (CMNPs) containing Ang-2 small interfering RNA (Ang2-siRNA) plasmids were developed, and these nanoparticles could lead to significant inhibition of angiogenesis in a malignant melanoma model. ${ }^{19}$ Magnetic iron oxide nanoparticles are the core of Ang2-CMNPs. The Ang2-siRNA plasmid particles were encapsulated by chitosan, which does not increase systemic toxicity and is stable in vivo. ${ }^{27}$ Additionally, the combination of the Ang2-siRNA system and the magnetic targeted drug delivery system can improve the function of siRNAs in vivo. Moreover, when the siRNA nucleic acid transfer system is 
A

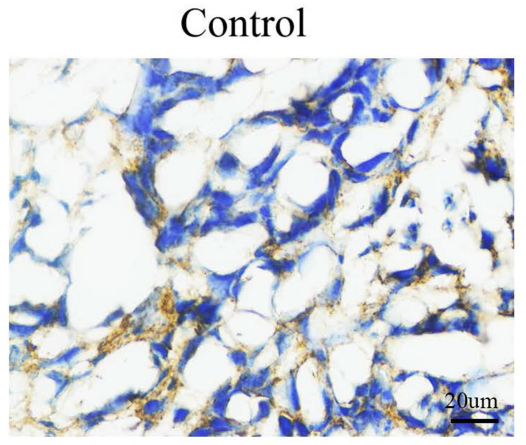

B

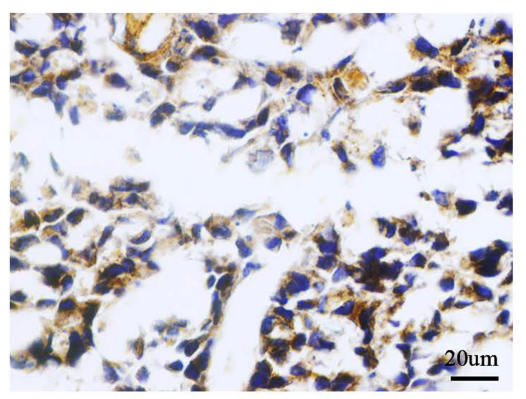

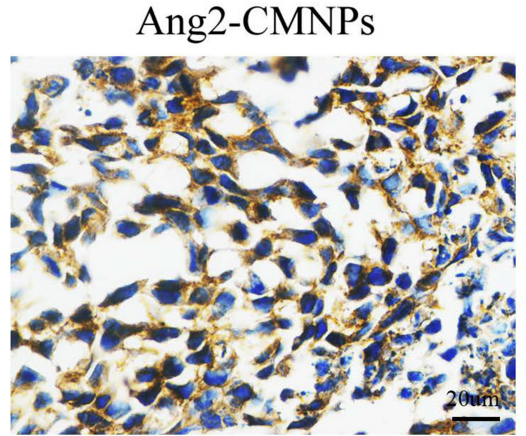

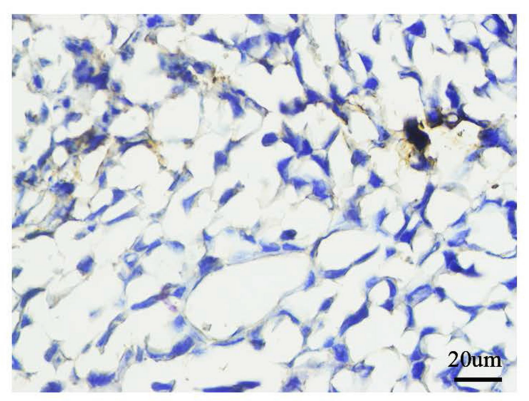

C
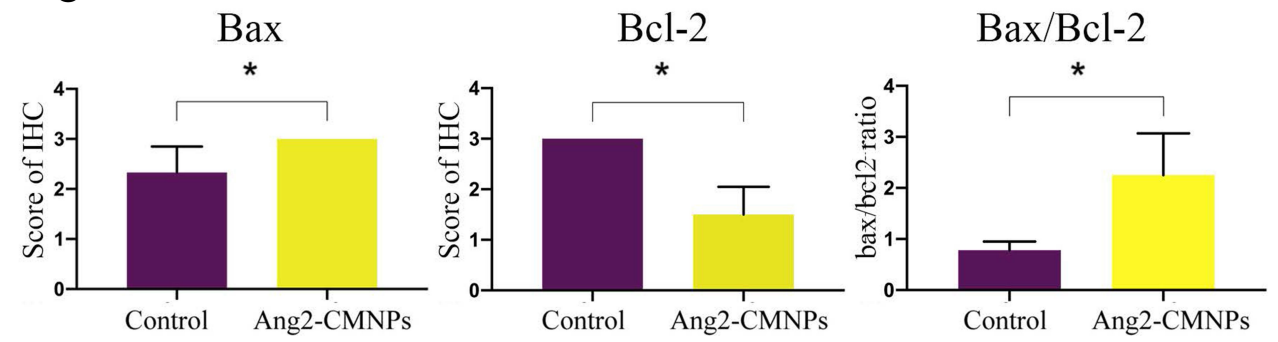

Figure 6 Ang2-CMNPs induced apoptosis through the mitochondria-mediated pathway. (A) Representative immunohistochemical images of the proapoptotic protein bax and $(\mathbf{B})$ the antiapoptotic protein Bcl-2 (400x) and (C) quantification of the Bax and Bcl-2 scores and their respective ratios. $* P<0.05$ compared with the control group.

used in combination with the chitosan magnetic nanoparticles, the serum pharmacokinetics of the targeted drug delivery system and the half-life of the composite nanoparticles can prolong the siRNA interference effect, which may result in lasting RNA interference effects and enhance the efficacy of gene interference treatment. ${ }^{28}$ Our previous study also showed that Ang2-CMNPs significantly inhibited the growth of malignant melanoma in vivo. ${ }^{19}$

We further evaluated the underlying mechanism of Ang2-CMNP-mediated inhibition of tumor growth. Angiogenesis occurs throughout tumor development and metastasis, and Ang2 has been shown to be essential during the proangiogenic and aggressive phases. In this study, we observed that the gene expression of Ang2 in malignant melanoma could be inhibited by Ang2-Ang2 in malignant melanoma could be inhibited by Ang2CMNPs. The Ang2-CMNPs were administered to malignant melanoma tissue to evaluate whether a single dose would be sufficient to induce a therapeutic effect. Our results showed that a single-dose of Ang2-CMNPs significantly inhibited malignant melanoma growth, decreased blood vessel formation, and increased normalappearing vessel regression. Given that inducing apoptosis in tumor cells is often accompanied by antitumor vasculature treatments, it is important to examine the apoptosis rate of melanoma cells. The TUNEL assay results revealed that the apoptosis rate of malignant melanoma cells in the Ang2-CMNP treatment group was significantly higher than that of the control group. However, the TUNEL assay results showed significantly increased tumor cell apoptosis in the Ang2-CMNP treatment group, and a decrease in the MVD could also lead to tumor cell necrosis. A previous study reported that Ang2 could target coopted vessels for regression via an apoptotic mechanism that may involve disrupted interactions between tumors and metastases. ${ }^{29}$ 
A

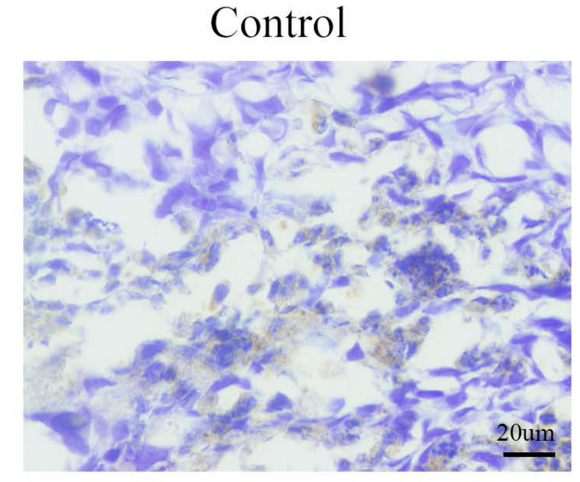

Ang2-CMNPs

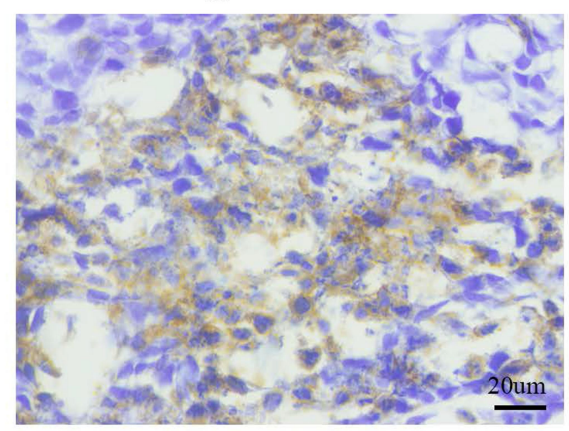

B

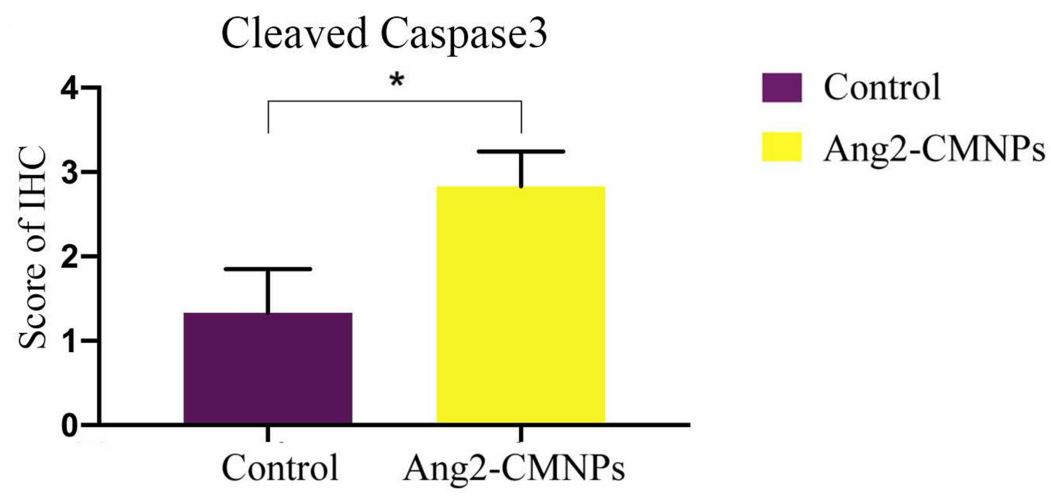

Figure 7 Ang2-CMNPs increased caspase 3 activity. (A) Cleaved caspase 3-positive cells show increased staining in the tumor tissue of the Ang2-CMNP-treated group compared with the empty magnetic nanoparticle-treated control group. (B) The immunohistochemistry scores based on cleaved caspase- 3 staining. $* P<0.05$ compared with the control group.

Promoting tumor cell apoptosis has been the main goal of anticancer drug development in the field of oncology for decades. The process of programmed cell death is mediated into two major pathways, the death receptor pathway and the mitochondrial pathway. ${ }^{30}$ The Bcl-2 and caspase protein families play crucial roles in the development of apoptosis. Our results demonstrated significantly increased expression levels of the proapoptotic protein Bax and decreased expression of the antiapoptotic protein Bcl-2, which were associated with changes in the mitochondrial membrane potential and the release of cytochrome C. ${ }^{31,32}$ Caspase- 3 , the downstream effector of cytochrome $\mathrm{C}$ and caspase- 9 , has been considered to be the key enzyme in the mitochondria-dependent apoptosis pathway. ${ }^{33}$ In this study, the expression levels of cleaved caspase 3 were significantly increased after treatment with Ang2-CMNPs, indicating that Ang2-CMNPs induced apoptosis through the mitochondrial-mediated pathway.

\section{Conclusion}

In summary, we evaluated the antitumor effects of Ang2CMNPs on malignant melanoma in nude mice and found that
Ang2-CMNP treatment increased the regression of normalappearing vessels regression in the tumor microenvironment and induced melanoma cell apoptosis through the mitochondrial-mediated pathway. Our study demonstrated that Ang2CMNPs could potentially serve as an effective treatment strategy to suppress malignant melanoma. Future studies are still needed to examine the potential of the downstream target gene of Ang2 and their efficacy in inhibiting the growth in malignant melanoma-bearing nude mice.

\section{Funding}

This study was funded by the High Level Hospital Maxillofacial Medical Center Funding; Projects of Fujian Provincial Natural Science Foundation (grant no. 2018J01163, 2018J01154); Scientific Research Foundation of National Health Planning Scientific Research FoundationJoint Research Projects of Fujian Provincial Health and Education (2019-WJ-09); Medical Innovation Project of Fujian Province (grant no. 2018-CX-26); National Natural Science Foundation of China (grant no. 81801931) and Fujian Province Science and Technology Innovation Joint Fund Project (grant no. 2019Y9114). 


\section{Disclosure}

The authors report no conflicts of interest in this work.

\section{References}

1. Siegel RL, Miller KD, Jemal A. Cancer statistics, 2019. CA Cancer J Clin. 2019;69(1):7-34. doi:10.3322/caac.21551

2. Dirk S, vAAC J, Carola B, et al. Melanoma. The Lancet. 2018.

3. Amm E, Blank CU, Mandala M, et al. Adjuvant pembrolizumab versus placebo in resected Stage III Melanoma. 2018;378:19.

4. S Y, L H, W Q, X R. Targeting angiogenesis by blocking the ATM-SerRS-VEGFA pathway for UV-induced skin photodamage and melanoma growth. Cancers. 2019;11(12).

5. Ferrara N, Adamis AP. Ten years of anti-vascular endothelial growth factor therapy. Nat Rev Drug Discov. 2016;15(6):385-403. doi:10.1038/nrd.2015.17

6. Kabbinavar F, Hurwitz HI, Fehrenbacher L, et al. Phase II, randomized trial comparing bevacizumab plus Fluorouracil (FU)/Leucovorin (LV) With FU/LV alone in patients with metastatic colorectal cancer. J Clin Oncol. 2003;21(1):60-65. doi:10.1200/JCO.2003.10.066

7. Yang JC, Haworth L, Sherry RM, Hwu P, Rosenberg SA. A randomized trial of bevacizumab, an anti-vascular endothelial growth factor antibody, for metastatic renal cancer. $N$ Engl $J$ Med. 2003;349(5):427-434. doi:10.1056/NEJMoa021491

8. Meadows KL, Hurwitz HI. Anti-VEGF therapies in the clinic. Cold Spring Harb Perspect Med. 2012;2(10):a006577-a006577. doi:10.1101/cshperspect.a006577

9. De Palma MHD. The biology of personalized cancer medicine: facing individual complexities underlying hallmark capabilities. Mol Oncol. 2012;6(2):111-127. doi:10.1016/j.molonc.2012.01.011

10. Bergers G, Hanahan D. Modes of resistance to anti-angiogenic therapy. Nat Rev Cancer. 2008;8(8):592-603. doi:10.1038/nrc2442

11. Rigamonti N, Kadioglu E, Keklikoglou I, Wyser Rmili C, Leow Ching C, De Palma M. Role of Angiopoietin-2 in adaptive tumor resistance to VEGF signaling blockade. Cell Rep. 2014;8 (3):696-706. doi:10.1016/j.celrep.2014.06.059

12. Toshiyuki K, Yashiro M, Kasashima H, et al. Oligodendrocytes up-regulate the invasive activity of glioblastoma cells via the Angiopoietin-2 signaling pathway. Anticancer Res. 2019.

13. Schmittnaegel M, Rigamonti N, Kadioglu E, et al. Dual angiopoietin-2 and VEGFA inhibition elicits antitumor immunity that is enhanced by PD-1 checkpoint blockade. Sci Transl Med. 2017;9(385):eaak9670. doi:10.1126/scitranslmed.aak9670

14. Huang H, Bhat A, Woodnutt G, Lappe R. Targeting the ANGPTTIE2 pathway in malignancy. Nat Rev Cancer. 2010;10(8):575-585. doi: $10.1038 / \mathrm{nrc} 2894$

15. Lewis Claire E, Ferrara N. Multiple Effects of Angiopoietin-2 Blockade on Tumors. Cancer Cell. 2011;19(4):431-433. doi:10.1016/j.ccr.2011.03.016

16. Papadopoulos KP, Kelley RK, Tolcher AW, Razak ARA, Siu LL. A Phase I First-in-Human Study of Nesvacumab (REGN910), a fully human anti-angiopoietin-2 (ang2) monoclonal antibody, in patients with advanced solid tumors. Clin Cancer Res off J Am Assoc Cancer Res. 2015;22:6.

17. Shanmuganathan R, Edison T, LewisOscar F, Kumar P, Shanmugam S, Pugazhendhi A. Chitosan nanopolymers: an overview of drug delivery against cancer. Int $J$ Biol Macromol. 2019;130:727-736. doi:10.1016/j.ijbiomac.2019.02.060
18. Sathiyavimal S, Vasantharaj S, LewisOscar F, Pugazhendhi A, Subashkumar R. Biosynthesis and characterization of hydroxyapatite and its composite (hydroxyapatite-gelatin-chitosan-fibrin-bone ash) for bone tissue engineering applications. Int $J$ Biol Macromol. 2019;129:844-852. doi:10.1016/j.ijbiomac.2019.02.058

19. Shan XY, Xu TT, Liu ZL, et al. Targeting of angiopoietin 2-small interfering RNA plasmid/chitosan magnetic nanoparticles in a mouse model of malignant melanoma in vivo. Oncol Lett. 2017;14 (2):2320-2324. doi:10.3892/ol.2017.6443

20. Zeinali Sehrig F, Majidi S, Nikzamir N, Nikzamir N, Nikzamir M, Akbarzadeh A. Magnetic nanoparticles as potential candidates for biomedical and biological applications. Artif Cells Nanomed Biotechnol. 2016;1-10.

21. Zeng D, Deng S, Sang C, Zhao J, Chen T. Rational design of cancer-targeted selenadiazole derivative as efficient radiosensitizer for precise cancer therapy. Bioconjug Chem. 2018;29(6):2039-2049. doi:10.1021/acs.bioconjchem. 8 b00247

22. H Y, K M, EH H, et al. Resistance mechanisms to anti-angiogenic therapies in cancer. Front Oncol. 2020;10:221. doi:10.3389/ fonc. 2020.00221

23. Edatt L, Poyyakkara A, Raji GR, Ramachandran V, Kumar VBS. Role of sirtuins in tumor angiogenesis. Front Oncol. 2020. doi:10.3389/fonc. 2019.01516

24. Goede V, Coutelle O, Neuneier J, et al. Identification of serum angiopoietin-2 as a biomarker for clinical outcome of colorectal cancer patients treated with bevacizumab-containing therapy. 2010;103(9):1407-1414.

25. Kim S, Dobi E, Jary M, Monnien F, Borg C. Bifractionated CPT-11 with LV5FU2 infusion (FOLFIRI-3) in combination with bevacizumab: clinical outcomes in first-line metastatic colorectal cancers according to plasma angiopoietin-2 levels. BMC Cancer. 2013;13 (1):611. doi:10.1186/1471-2407-13-611

26. Wu X, Giobbie-Hurder A, Liao X, et al. Angiopoietin-2 as a biomarker and target for immune checkpoint therapy. Cancer Immunol Res. 2017;5(1):17-28. doi:10.1158/2326-6066.CIR-16-0206

27. Liu ZL, You CL, Wang B, Lin JH, Zhang YD. Construction of Ang2-siRNA chitosan magnetic nanoparticles and the effect on Ang2 gene expression in human malignant melanoma cells. Oncol Lett. 2016;11(6):3992-3998. doi:10.3892/ol.2016.4539

28. Scholz C, Wagner E. Therapeutic plasmid DNA versus siRNA delivery: common and different tasks for synthetic carriers. $J$ Controlled Rel. 2012;161(2). doi:10.1016/j.jconrel.2011.11.014

29. Holash J, Compton D, et al. Vessel cooption, regression, and growth in tumors mediated by angiopoietins and VEGF. Science. 1999;284 (5422):1994-1998. doi:10.1126/science.284.5422.1994

30. Denicourt C, Dowdy SF. Targeting apoptotic pathways in cancer cells. ence. 2004;305(5689):1411-1413. doi:10.1126/ science. 1102974

31. Carneiro BA, El-Deiry WS. Targeting apoptosis in cancer therapy. Nat Rev Clin Oncol. 2020.

32. Yan X, Zhang C, Ouyang J, et al. Atmospheric pressure plasma treatments protect neural cells from ischemic stroke-relevant injuries by targeting mitochondria. Plasma Process Polym. n/a;n/a:e2000063.

33. Bock FJ, Tait SWG. Mitochondria as multifaceted regulators of cell death. Nat Rev Mol Cell Biol. 2020;21(2):85-100. doi:10.1038/ s41580-019-0173-8 


\section{Publish your work in this journal}

Cancer Management and Research is an international, peer-reviewed open access journal focusing on cancer research and the optimal use of preventative and integrated treatment interventions to achieve improved outcomes, enhanced survival and quality of life for the cancer patient.

The manuscript management system is completely online and includes a very quick and fair peer-review system, which is all easy to use. Visit http://www.dovepress.com/testimonials.php to read real quotes from published authors.

Submit your manuscript here: https://www.dovepress.com/cancer-management-and-research-journal 